\title{
Scheduling bus holding times in time horizons considering transfer synchronization and service regularity
}

\author{
Jesse Voorhorst $^{1}$ and Konstantinos Gkiotsalitis ${ }^{2}$
}

\begin{abstract}
Transfers between public transport modes hinder the traveling by public transport due to the unreliability and long waiting times. Nonetheless, public transport operators are mainly interested in maintaining the regularity of different lines without accounting for the transfer waiting times. This work focuses on the application of bus holding to reduce the transfer waiting times, in-vehicle waiting times, and the deviation from the target headways. The determination of optimal holding times is performed in time horizons (e.g., hourly time periods) where past events are fixed, while future events are considered in the optimization process. This study models the bus holding problem which considers transfer synchronization as a nonlinear convex program (NLP) that can be solved by off-the-shelf solvers. Our approach is tested against state-of-the-art bus holding methods in a case study on the bus network of Almere (Netherlands) demonstrating a significant reduction in the transferring waiting times.
\end{abstract}

Keywords: scheduling holding times; transfer synchronization; bus regularity; nonlinear programming.

\section{INTRODUCTION}

Congestion and air pollution are major problems that can be reduced by an increased use of public transport. However, to increase the public transport ridership public transport services have to be attractive. Frequency, unreliability and long waiting times at transfers between lines are seen as barriers to using public transport [1], [2]. Several studies have shown that people desire short waiting times and easy transfers [3], [4]. Therefore, many public transport timetables include planned transfers to reduce the waiting times at the transfer stop(s) [5], [6]. Even if timetables consider the planned transfers to reduce the waiting times, operational delays might result in passengers missing their connection and, consequently, wait for a long time period. Because unreliability is one of the most important aspects in bus operations [7], [8], we consider the impact of operational delays to planned transfers, in-vehicle passenger waiting times, and waiting times of regular passengers at non-transfer stops.

Existing literature mostly focuses on optimization of transfers in the timetable development phase [9]. Fleurent et al. [10] use the concept of a "trip meet" to describe a good connection. A "trip meet" occurs if the waiting time is not too long or too short. They suggest a minimum of 3 minutes and a maximum of 10 minutes for these boundaries. Furthermore,

${ }^{1}$ Jesse Voorhorst is with the Department of Civil Engineering, University of Twente, 7500 AE Enschede, The Netherlands j.b. voorhorstestudent.utwente.nl

${ }^{2}$ Konstantinos Gkiotsalitis is Assistant Professor at the Department of Civil Engineering, University of Twente, 7500 AE Enschede, The Netherlands k.gkiotsalitis@utwente.nl they weight these "trip meets" based on importance. Their optimization algorithm uses a "trip meet" quality index as objective, which is defined as the difference between the ideal waiting time and the actual waiting time as long as the waiting time is still within the admissible range. This objective was integrated in a global optimization algorithm which also considers the fleet size and deviation from the target headway. Ibarra-Rojas and Rios-Solis [11] also use trip meets, but their model just maximizes the number of successful synchronizations. In their work, headways are taken into account as a constraint, but fleet size is not taken into account.

$\mathrm{Wu}$ et al. [12] describe a model which optimizes the waiting time of transfer passengers in the Beijing subway and tries to distribute the waiting time over multiple transfer stations using a min-max function. This formulation treats the regularity of the lines as a constraint and vehicle scheduling is not taken into account. Parbo et al. [13] apply a similar technique. Their objective function only consists out of the waiting times at transfer stops multiplied by the number of passengers transferring and their value of time split into different passenger groups. Only the offset at the first stop of an existing timetable can be changed, and their model considers changes to the passenger numbers as a result of the new timetable.

A planning model which includes transfers, regularity and fleet size is [14]. Their bi-objective, bi-level optimizes the total operation costs based on the fleet size, the total passenger initial waiting time, total passenger transfer waiting time and the overcrowding hours. The last three aspects are combined into one in the objective function, alongside the fleet size.

Other works created models which re-optimize the timetable during the actual operations. In this research area, most works focus on maintaining the regularity or headways, e.g. [15], [16], [17]. The aforementioned works solve the bus holding and rescheduling problem with heuristic algorithms based on real-time vehicle location information to increase the regularity of the lines. In Nesheli and Ceder [18], passenger transfers are taken into consideration as a potential reason for holding vehicles. The regularity is not included directly, but is maintained by minimizing the waiting time of passengers at stops further downstream. Furthermore, besides holding, skip-stopping is also included as a potential measure. Their model can decide which control action is most suitable in a specific situation, although it is a deterministic model which, for instance, assumes that the future passenger demand is not varying. Their objective aims 
to minimize the total additional waiting time for passengers during their transfer, when they are already in the bus, or waiting at a stop downstream. Nesheli and Ceder [19] is an extension to [18] considering the reliability of the network and the sensitivity of certain variables to control measures. Nesheli et al. [20] extend the two previous works further by including a short-turning strategy via using a variable indicating if a direct transfer is possible.

Gkiotsalitis and Maskelar [21] reschedule trips and optimize the expected waiting time and transfer waiting time using a sequential hill-climbing algorithm. The expected waiting time is an alternative way to include the headway deviation in the optimization model. Their model does not take the vehicle allocation into account, which can lead to the need of more vehicles to be able to operate the re-optimised timetable. The model of [22] is specifically created for rail systems and minimizes the waiting time of transferring passengers. The regularity is not included as objective, but headways are constrained. Dwell times are treated as a decision variable and are constrained as well.

In the literature, there are multiple works that minimize the waiting time of transferring passengers - but mainly during the timetable planning phase (see [10], [13], [14], [18], [20], [21], [22]). Most of them also consider the regularity by minimising the expected waiting time of passengers. However, some studies that re-optimise the timetable during operation (also known as rescheduling) only include a maximum or minimum headway as problem constraint. Additionally, most models do not take the vehicle scheduling, and thus the vehicle availability, into account. Furthermore, in past literature rescheduling typically changes the dispatching times of trips without devising bus holding strategies.

This paper considers periodic re-optimization of a public transport network by scheduling new bus holding measures. Besides minimizing the waiting times of transferring passengers, the regularity of individual bus lines is also part of the objective function. In this study, the regularity is defined as the deviation between the actual and the target headway. Furthermore, extra waiting times at stops downstream of the holding stop are included. These three aspects are combined with different weights, which makes it possible to change the optimization focus depending on priorities in the specific area. Existing research using real-time or rolling horizon models does not take vehicle availability constraints into account, while this work considers their impact on the bus holding options. Furthermore, we consider both the transferring waiting times and the in-vehicle travel times, which, in most studies, is not taken into account.

\section{PROBLEM FORMULATION}

Travel time variations during the actual operations are the main factor of irregular services and missed passenger transfers [23], [24], [25]. To rectify this, we propose bus holding schedules which can be updated in time horizons (e.g., every hour).

This work makes a number of reasonable assumptions, such as: (i) the number of bus trips per line is decided during the frequency settings phase and all of the assigned trips are expected to be performed during the day [26]; and (ii) bus trips from the same line are not expected to overtake one another (an assumption used in several operational planning works, such as [15], [27]).

The notation of our multi-line synchronization problem in time horizons is presented in Table I.

TABLE I

NOMENCLATURE

\section{Sets}

$L \quad L=\{1, \ldots, l, \ldots\}$ are the different bus lines in the study area

$N(l) \quad N(l)=\{1, \ldots, n, \ldots\}$ is the set of trips of each bus line $l \in L$ that are planned to be dispatched during the time horizon where we schedule the holding times

$S(l) \quad S(l)=\{1, \ldots, s, \ldots\}$ is the set of bus stops of each bus line $l \in L$ ordered from the first to the last

$B_{l j} \quad$ all transfer stops between lines $l$ and $j$ where the arrival times of trips that belong to line $l$ need to be synchronized with the arrival times of trips that belong to line $j$

\section{Parameters}

$f_{l} \quad$ is the number of trips for each line $l \in L$ which are needed to fulfill the demand (note: the number of trips is already determined at the frequency settings stage)

$T$ the planning period (note: the suggested planning period is at most one day of operations)

$h_{l}^{*} \quad h_{l}^{*}=\frac{T}{f_{l}}$ is the ideal headway of bus line $l \in L$ that should be maintained at all bus stops for attaining a perfectly regular service $(\mathrm{sec})$

$t_{l, n, s} \quad$ denotes the expected travel time of bus trip $n$ of line $l$ between stops $s-1$ and $s$ (sec)

$\delta_{l}^{\min } \quad$ is the dispatching time of the first trip of the planning period ( $\mathrm{sec})$

$\delta_{l}^{\max } \quad$ is the latest possible time where all trips of line $l \in L$ must have completed their service for preventing schedule sliding $(\mathrm{sec})$

$k_{l, n, s} \quad$ is the expected dwell time of bus trip $n$ of bus line $l$ at stop $s$ $(\mathrm{sec})$

$\psi_{l} \quad$ is the required layover time for line $l$ after completing each bus trip (sec)

$d_{l, n} \quad$ the scheduled dispatching time of trip $n$ that belongs to line $l$

$a_{1} \quad$ weight of waiting time of transferring passengers

$a_{2} \quad$ weight of the extra travel time due to bus holdings

$a_{3} \quad$ weight of the service regularity, measured as the deviation of the inter-arrival headways from their scheduled values

$\pi_{l j}^{b n m} \quad$ transfer demand weight based on the number of expected passengers willing to transfer from trip $n$ of line $l$ to trip $j$ of line $m$ at transfer stop $b$

$\pi_{l, n, s} \quad$ in-vehicle demand weight based on the number of in-vehicle passengers of trip $n$ of line $l$ at stop $s$

$\psi_{l} \quad$ required layover time for bus line $l$

$\Phi_{n, n^{\prime}}^{l} \quad \Phi_{n, n^{\prime}}^{l}=1$ if trip $n^{\prime} \in N(l)$ is the following trip of trip $n$ that is operated by the same bus, and zero otherwise

$Y_{l j}^{b n m} \quad Y_{l j}^{b n m}=1$ is trip $n \in N(l)$ needs to synchronize its arrival time with trip $m \in N(j)$ at transfer stop $b \in B_{l j}$, and zero otherwise

$H_{l}^{\min } \quad$ minimum allowed headway of successive vehicles of line $l$

$H_{l}^{\max }$ maximum allowed headway of successive vehicles of line $l$

\section{Variables}

$x_{l, n, s} \quad$ is the bus holding time of the $n^{\text {th }}$ trip that belongs to line $l$ at stop $s(\mathrm{sec})$. Note that this is the decision variable of our problem $a_{l, n, s} \quad$ the arrival time of trip $n$ that belongs to line $l$ at stop $s$

$h_{l, n, s} \quad$ the inter-arrival headway between trips $n, n-1$ that belong to line $l$ at stop $s$

Following the notation in Table I, the arrival time of any trip $n$ that belongs to a bus line $l \in L$ at stop $s \in S(l) \backslash\{1\}$ is: 


$$
a_{l, n, s}=d_{l, n}+\sum_{z=2}^{s} t_{l, n, z}+\sum_{z=1}^{s-1}\left(k_{l, n, z}+x_{l, n, z}\right)
$$

In Eq. 1 the arrival time of a trip $n$ at stop $s$ is set equal to the departure time of the trip, $d_{l, n}$, plus the sum of the expected travel times between consecutive stops until reaching stop $s, \sum_{z=2}^{s} t_{l, n, z}$, plus the expected dwell time at each bus stop until reaching stop $s, \sum_{z=1}^{s-1} k_{l, n, z}$, plus the scheduled holding times at each bus stop until reaching stop $s, \sum_{z=1}^{s-1} x_{l, n, z}$.

\section{A. Objective Function}

To increase the regularity of bus services, the actual interarrival time headways at bus stops should be as close as possible to their scheduled values, $h_{l}^{*}=\frac{T}{f_{l}}$, for each line $l \in$ $L$. The inter-arrival time headway between two consecutive services $n-1, n$ of line $l$ at stop $s$ is:

$$
h_{l, n, s}=a_{l, n, s}-a_{l, n-1, s} \text { where } n \in N(l) \backslash\{1\}
$$

The difference between the actual headways and the ideal headways at stops is the sole key performance indicator of regularity-based services and has been in use in London, Singapore, Barcelona and many other densely populated areas where the bus services operate in high frequencies [28]. To reduce the difference between the actual inter-arrival headways and the ideal ones for a bus line $l \in L$, one should minimize the sum of the squared difference between the actual and the ideal headways (see [29]):

$$
\sum_{s \in S(l)} \sum_{l \in L} \sum_{n \in N(l) \backslash\{1\}}\left(h_{l, n, s}-h_{l}^{*}\right)^{2}
$$

Apart from the service regularity, we also consider the additional travel time because of the imposed holding times. This can be expressed as:

$$
\sum_{l \in L} \sum_{s \in S(l) \backslash\{1\}} \sum_{n \in N(l)} \pi_{l, n, s} x_{l, n, s}
$$

By minimizing Eq.4, we strive to reduce the time period of the imposed headways because they increase the trip travel times of in-vehicle passengers. Note that $x_{l, n, s}$ is multiplied by the in-vehicle demand weight $\pi_{l, n, s}$ because holding a vehicle with more passengers onboard results in increased inconvenience [30].

Last, we also consider the waiting times of passengers that miss their transfer connection. Let $Y_{l j}^{b n m}$ be a binary parameter where $Y_{l j}^{b n m}=1$ if trip $n \in N(l)$ needs to synchronize its arrival time with trip $m \in N(j)$ at the transfer stop $b \in B_{l j}$ and $Y_{l j}^{b n m}=0$ otherwise. Ceder et al. [6] considers a perfect synchronization when trip $n$ arrives at the transfer stop $b \in B_{l j}$ exactly at the same time as trip $m \in N(j)$. In this way, the waiting times of passengers that want to transfer from bus trip $n \in N(l)$ to bus trip $m \in N(j)$ at bus stop $b \in B_{l j}$ are minimized when $a_{l, n, b}-a_{j, m, b}=0$.

In our study, we follow the definition of [6] and we consider a synchronization of passengers of trip $n \in N(l)$ who transfer to trip $n \in N(j)$ when

$$
\begin{aligned}
Y_{l j}^{b n m} & \left(a_{l, n, b}-a_{j, m, b}\right)=0 \\
& \forall l, j \in L, \forall n \in N(l), \forall m \in N(j) \backslash\{1\}, \forall b \in B_{l j}
\end{aligned}
$$

Note that when $Y_{l j}^{b n m}=0$ the inequalities of Eq.5 hold for any value of the arrival times $a_{l, n, b}$ and $a_{j, m, b}$ because in such case there is no requirement for synchronization. To penalize the increase of transfer waiting times in case of a missed synchronization, we need to minimize the transfer waiting times of all passengers in our time horizon:

$$
\sum_{l \in L} \sum_{j \in L \backslash\{l\}} \sum_{n \in N(l)} \sum_{m \in N(j) \backslash\{1\}} \sum_{b \in B_{l j}} \pi_{l j}^{b n m} Y_{l j}^{b n m}\left(a_{l, n, b}-a_{j, m, b}\right)
$$

where the transfer demand weight $\pi_{l j}^{b n m}$ is used to place more importance on synchronized transfers with increased passenger demand.

Combining Eq.3,4 and 6 yields our objective function:

$$
f(x, h, a):=
$$

$$
\begin{gathered}
a_{1} \sum_{l \in L} \sum_{j \in L \backslash\{l\}} \sum_{n \in N(l)} \sum_{m \in N(j) \backslash\{1\}} \sum_{b \in B_{l j}} \pi_{l j}^{b n m} Y_{l j}^{b n m}\left(a_{l, n, b}-a_{j, m, b}\right)+ \\
a_{2} \sum_{l \in L} \sum_{s \in S(l) \backslash\{1\}} \sum_{n \in N(l)} \pi_{l, n, s} x_{l, n, s}+ \\
\quad a_{3} \sum_{s \in S(l)} \sum_{l \in L} \sum_{n \in N(l) \backslash\{1\}}\left(h_{l, n, s}-h_{l}^{*}\right)^{2}
\end{gathered}
$$

where $a_{1}$ is a weight factor indicating the importance of transfer waiting times, $a_{2}$ a weight factor indicating the importance of extra in-vehicle travel times due to bus holdings, and $a_{3}$ a weight factor indicating the importance of irregularities in the service.

\section{B. Vehicle circulation and schedule sliding constraints}

Reckon that $\Phi_{n, n^{\prime}}^{l}=1$ if bus trip $n^{\prime} \in N(l)$ is operated after the completion of bus trip $n \in N(l)$ by the same bus and $\Phi_{n, n^{\prime}}^{l}=0$ otherwise. Then, if the required layover time for bus line $l \in L$ is $\psi_{l}$, the holding times, $x_{l, n^{\prime}, s}$, of trip $n^{\prime}$ should satisfy the inequality:

$$
\begin{array}{r}
\Phi_{n, n^{\prime}}^{l}\left(d_{l, n^{\prime}}-\left(d_{l, n}+\sum_{s \in S(l) \backslash\{1\}} t_{l, n, z}+\sum_{s \in S(l)}\left(k_{l, n, z}+x_{l, n, z}\right)\right)\right) \geq \\
\Phi_{n, n^{\prime}}^{l} \psi_{l} \quad, \quad \forall n, n^{\prime} \in N(l), \forall l \in L \quad \text { (8) }
\end{array}
$$

Note that the inequality of Eq.8 is always satisfied when bus trip $n^{\prime}$ is not operated by the same bus as trip $n$ because then $\Phi_{n, n^{\prime}}^{l}=0$ and the inequality holds.

Finally, to prevent schedule sliding and maintain the duration of the rolling horizon, all trips of any bus line $l \in L$ must have been completed before time $\delta_{l}^{\text {max }}$. Avoiding schedule sliding yields the following inequality constraints:

$$
\begin{aligned}
d_{l, n}+\sum_{s \in S(l) \backslash\{1\}} t_{l, n, z}+\sum_{s \in S(l)}\left(k_{l, n, z}+x_{l, n, z}\right) & \leq \delta_{l}^{\max } \\
\forall n & \in N(l), l \in L
\end{aligned}
$$


which ensures that each trip $n$ of line $l$ has arrived at the last stop and has completed all passenger alightings before time $\delta_{l}^{\max }$.

\section{Mathematical Program of the Network-Wide SYNCHRONIZATION PROBLEM}

Our bus holding synchronization model explicitly considers the regularity of the bus lines, the transfer synchronizations, and the in-vehicle travel time increase due to bus holdings:

$$
\begin{array}{ll}
(Q): \min _{x, h, a} & f(x, h, a) \\
\text { s.t.: } & H_{l}^{\text {min }} \leq h_{l, n, s} \leq H_{l}^{\max }, \forall l \in L, n \in N(l), s \in S(l) \\
& (x, a, h) \in \mathscr{F}=\{(x, a, h) \text { satisfy Eq. } 1-2,8-9\} \\
& x_{l, n, s} \in \mathbb{R}_{\geq 0} \quad, \quad \forall l \in L, \forall n \in N(l), \forall s \in S(l)
\end{array}
$$

where constraints $H_{l}^{\min } \leq h_{l, n, s} \leq H_{l}^{\max }, \quad \forall l \in L, n \in$ $N(l), \quad s \in S(l)$ ensure that the minimum and maximum allowed headways of successive vehicles of any line $l \in L$ are met.

Program $(Q)$ is a convex nonlinear optimization problem and can be solved to global optimality with a solution method for nonlinear programming (e.g., the interior point method [31] available in many off-the-shelf solvers). The formal proof is provided in Theorem 3.1.

Theorem 3.1: Program $(Q)$ is a convex optimization problem.

Proof: The constraints of our optimization problem are linear (in)equalities. Ergo, the feasible region $\mathscr{F}$ is a polyhedron; and thus, a convex set. That is, it is sufficient to prove that the objective function $f(x, h, a)$ is convex.

$f(x, h, a)$ is the sum of two linear convex functions and a quadratic convex function. Thus, $f(x, h, a)$ is also convex as the sum of convex functions (see [32]) and this completes our proof.

\section{CASE STUDY}

\section{A. Case description}

Our case study is the city of Almere in the Netherlands. The network exists of 7 frequent lines which are marketed as high frequency routes (M1 to M7), 5 regional bus routes from suburbs to Amsterdam (322, 323, 326, 327 and 328), a local bus route to a neighborhood under development (25/525), and a bus on demand to an industrial estate (22) operated under the name AllGo. Besides these, there are 2 regional bus routes from the centre of Almere to the towns of Zeewolde and Harderwijk with a low frequency.

In our case study, we simplify the public transport system to form a self-contained network. The regional routes with low frequency are removed as travelling within Almere is not allowed on these routes. Two regional routes between the Parkwijk neighborhood of Almere and Amsterdam (322 and 323) connect many neighborhoods within Almere and are in some cases the only routes that serve a neighborhood.
Therefore, these are included in the simplified model, but are modeled to start and end at the first stop outside Almere, in Muiden. Furthermore, these are modeled as one route as the route within Almere is fully shared and route 323 does not operate outside peak hours. Routes 326 to 328 are excluded from the model as these connect Almere Haven neighborhood directly to Amsterdam and a local route runs parallel to it, so local journeys will be made with the local route. Route 22 serves different bus stops depending on demand which makes it unsuitable for this model. The simplified bus network is shown in Fig.1.

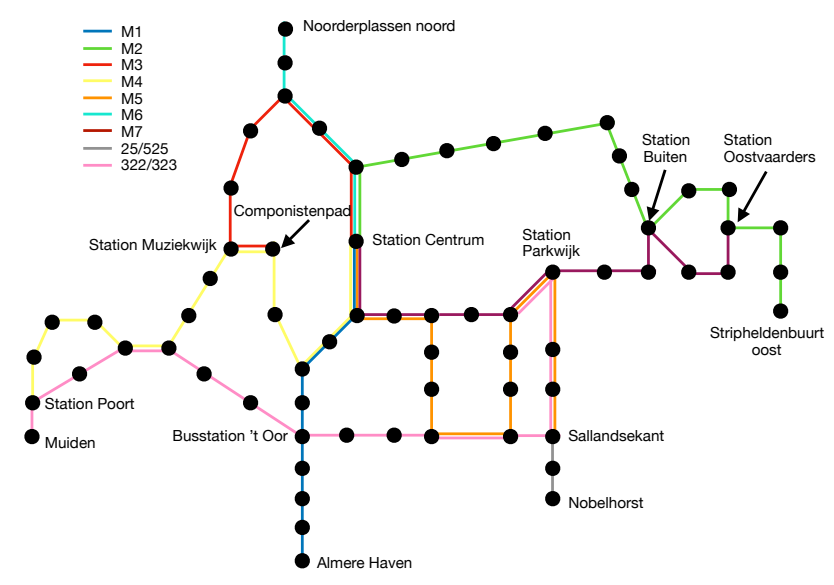

Fig. 1. Simplified bus network of Almere with stop names of end points and most important stops.

In this case study, we consider an hourly time horizon from 10:15 to $11: 15$ to plan our bus holding schedule. This time horizon is selected because it is at the morning peak. The routes were split into separate routes for both directions. Their frequencies in this time horizon are presented in Table II. The capacity of the buses is 89 passengers, including standees. The capacity of route $25 / 525$ is 8 passengers because it is operated by small buses.

Expected travel times and planned departure times at the first stops were obtained from the original timetable (see [33]). Minimum headways, $H_{l}^{\text {min }}$, were 2 minutes for the most frequent routes, 4 minutes for M6, 5 minutes for 25/525 and 10 minutes for route $322 / 323$. The maximum headways, $H_{l}^{\max }$, are 20,25, 35 and 65 minutes for the aforementioned categories, respectively. The boarding time is approximately 3 seconds per passenger and the alighting time 2 seconds, as most passengers are using a smart card. The maximum allowed slack time of each trip is based on the difference between the arrival of the trip and departure of the next trip in the opposite direction. Vehicles operating on routes M1 and M2 start a trip on the other route upon arrival at Station Centrum, which was also taken into account. Transfer times between routes at a stop are found by digitally measuring the walking distance based on an average walking speed of $4 \mathrm{~km} / \mathrm{h}$.

At the original timetables, there is no planned holding. All planned arrivals are obtained from the timetable [33]. 
TABLE II

OVERVIEW OF ROUTES AND FREQUENCIES [33]

\begin{tabular}{rrrr}
\hline Route nr & Origin & Destination & Frequency \\
\hline M1 & Station Centrum & Almere Haven & $8 \mathrm{trips} / \mathrm{h}$ \\
M2 & Station Centrum & Stripheldenbuurt Oost & $8 \mathrm{trips} / \mathrm{h}$ \\
M3 & Station Centrum & Componistenpad & $8 \mathrm{trips} / \mathrm{h}$ \\
M4 & Station Centrum & Station Poort & $8 \mathrm{trips} / \mathrm{h}$ \\
M5 & Station Centrum & Sallandsekant & $8 \mathrm{trips} / \mathrm{h}$ \\
M6 & Station Centrum & Noorderplassen Noord & $6 \mathrm{trips} / \mathrm{h}$ \\
M7 & Station Centrum & Station Oostvaarders & $8 \mathrm{trips} / \mathrm{h}$ \\
$\mathbf{2 5 / 5 2 5}$ & Sallandsekant & Nobelhorst & $4 \mathrm{trips} / \mathrm{h}$ \\
$\mathbf{3 2 2} / \mathbf{3 2 3}$ & stad Parkwijk & Bijlmer Arena & $2 \mathrm{trips} / \mathrm{h}$ \\
\hline
\end{tabular}

The average maximum number of passengers in a vehicle during a trip is approximately 30 to 40 .

\section{B. Sensitivity analysis and performance comparison against state-of-the-art methods}

The weights $a_{1}, a_{2}, a_{3}$ provide the possibility to change the importance of the three parts that form our objective function. This makes it possible to change the importance of different factors (e.g., transfer waiting times, in-vehicle travel times) based on the specific situation of the application area. Besides, it can be used for comparison with past works which use similar models. Table III shows the results of applying our bus holding schedule and other bus holding schedules derived by past studies. As a reference, the results per part of the objective function are provided in case of no holding, i.e., the initial solution of the scheduled timetable.

TABLE III

RESULTS USING DIFFERENT WEIGHTS IN THE OBJECTIVE FUNCTION AND COMPARISON AGAINST STATE-OF-THE-ART MODELS

\begin{tabular}{|c|c|c|c|c|c|c|c|}
\hline & \multirow[t]{2}{*}{$a_{1}$} & \multirow[t]{2}{*}{$a_{2}$} & \multirow[t]{2}{*}{$a_{3}$} & \multicolumn{4}{|c|}{ Objective function value } \\
\hline & & & & $\begin{array}{l}\text { transfer } \\
\text { waiting }\end{array}$ & $\begin{array}{l}\text { extra in-veh. } \\
\text { travel time }\end{array}$ & $\begin{array}{r}\text { service } \\
\text { regularity }\end{array}$ & overall \\
\hline No holding & 0 & 0 & 0 & 5755.7 & 0 & 6794.3 & $\mathrm{n} / \mathrm{a}$ \\
\hline Wong et al. [22] & 1 & 0 & 0 & 5675.8 & 181.78 & 6858.3 & 5675.8 \\
\hline \multirow{3}{*}{ Chen et al. [15] } & 0 & 0.2 & 0.8 & 5781.9 & 47.724 & 6658.9 & 5336.7 \\
\hline & 0 & 1 & 0.28 & 5757.7 & 11.132 & 6690.4 & 1884.4 \\
\hline & 0 & 0 & 1 & 5749.6 & 97.465 & 6566 & 6566 \\
\hline \multirow{11}{*}{ Our approach } & 0.3 & 0.2 & 0.5 & 5744.9 & 54.532 & 6548.4 & 5008.6 \\
\hline & 0.4 & 0.2 & 0.4 & 5724.8 & 60.241 & 6680.1 & 4974 \\
\hline & 0.5 & 0.2 & 0.3 & 5732.8 & 36.461 & 6694.7 & 4882.1 \\
\hline & 0.6 & 0.2 & 0.2 & 5729 & 30.507 & 6677.6 & 4779 \\
\hline & 0.7 & 0.2 & 0.1 & 5727.3 & 35.246 & 6750.2 & 4691.2 \\
\hline & 0.8 & 0.2 & 0 & 5701.2 & 36.707 & 6818.2 & 4568.3 \\
\hline & 0.2 & 0.2 & 0.6 & 5739.9 & 54.583 & 6673.5 & 5163 \\
\hline & 0.1 & 0.2 & 0.7 & 5747.3 & 62.486 & 6587.7 & 5198.6 \\
\hline & 0.3 & 0 & 0.7 & 5717.9 & 104.56 & 6632.3 & 6358 \\
\hline & 0.5 & 0 & 0.5 & 5712.3 & 104.66 & 6646.1 & 6179.2 \\
\hline & 0.4 & 0 & 0.6 & 5747.4 & 98.142 & 6645 & 6286 \\
\hline
\end{tabular}

In Table III, we present the results after comparing our model against against:

- the do-nothing case of no holding (scheduled timetable)

- the model of Wong et al. [22] that optimizes only the waiting times of transferring passengers (that is, $a_{2}, a_{3}=$ $0)$.

- the model of Chen et al. [15] that optimizes the invehicle travel times of passengers and the service regularity, while ignoring the travel times of transferring passengers (that is, $a_{1}=0$ ).
In our performance evaluation, we investigate the performance of our model for different values of the weight factors $a_{1}, a_{2}, a_{3}$, as it is detailed in Table III. The first mentioned configuration is used as a base case, having a weight of 0.3 for transfer waiting time, 0.2 for vehicle waiting and 0.5 for regularity. It becomes clear that a higher weight for one of the objective function components generally leads to a reduction of the objective value associated with that component. The transfer waiting time objective value does not differ much between configurations, the lowest being 5701 and the highest being 5747 .

The base case of $a_{1}=0.3, a_{2}=0.2, a_{3}=0.5$ performs better on both the transfer waiting time and regularity objective values compared to a situation without any holding, although the difference for the transfer waiting time is limited. No "overall objective value" is provided for the case of no holding because weights are required to evaluate the objective function. As expected, our model performs worse than the model of [22] in terms of transferring waiting times, as their model only regards the transfer waiting time. Nonetheless, our model performs better in terms of the invehicle waiting time (reduces from 182 to 54 in the base case or 31 in the best configuration). Additionally, the regularity improves in every case when using our model (especially in the base case).

Comparing the results with possible configurations based on Chen et al. [15], the most interesting observation is that the regularity in our base case performs better on the regularity than the situation with a full focus on regularity. The transfer waiting time also improves, but to a limited extent. The in-vehicle waiting time is better when focusing only on the regularity, but not comparable with the case where the in-vehicle waiting time is the only objective of the model.

Fig. 2 graphically shows the influence of the weight of the transfer waiting time component of the objective function on its value and the value of the regularity component. Our base configuration has the best value for the regularity and one of the best for the transfer waiting time component. The removal of the in-vehicle waiting time weight results in a reduction of transfer waiting time of about 30 passenger minutes, but regularity becomes worse than before.

\section{CONCLUSIONS}

The presented model optimizes a combination of the waiting time of transferring passengers, extra traveling times of passengers in a vehicle, and the regularity of a route in one objective function, capable of changing priorities based on local circumstances. The application on the network of Almere (Netherlands) shows that our model decreases the deviation between headways and target headways and, at the same time, improves the transfer waiting times of passengers. This comes at the cost of extra traveling times for the passengers already in the vehicle.

Our model has a better performance on both the transfer waiting times and service regularity than the model of Chen et al. [15] that only considered regularity and in-vehicle 


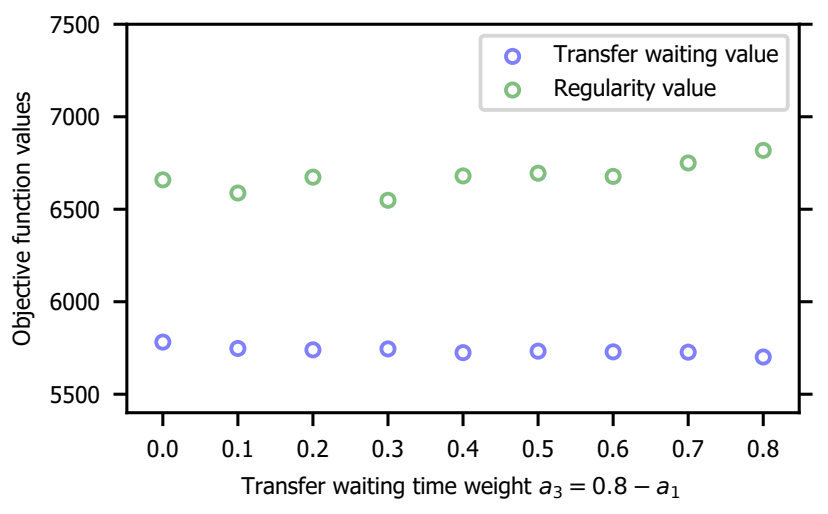

Fig. 2. Average objective function value with different transfer waiting time and regularity weights and a constant in-vehicle waiting time weight $a_{2}=0.2$.

waiting times. Compared to only considering transfer waiting time, e.g., Wong et al. [22], the transfer waiting time is higher, but the regularity is better. This shows that the model with the default weights does provide a balance between the different components of the objective function.

Main limitations of our approach are that the passenger arrival rates are assumed constant, despite that in reality this may not be always the case. However, the use of short time horizons when rescheduling the holding times can mitigate the effects of this problem. Considering future research, our model can be expanded with probabilistic travel times and passenger numbers to take into consideration the stochastic nature of these parameters. Another possible expansion is enabling different boarding and alighting doors in the algorithm.

\section{REFERENCES}

[1] G. Beirão and J. S. Cabral, "Understanding attitudes towards public transport and private car: A qualitative study," Transport policy, vol. 14, no. 6, pp. 478-489, 2007.

[2] L. Redman, M. Friman, T. Gärling, and T. Hartig, "Quality attributes of public transport that attract car users: A research review," Transport policy, vol. 25, pp. 119-127, 2013.

[3] L. DellOlio, A. Ibeas, and P. Cecin, "The quality of service desired by public transport users," Transport Policy, vol. 18, no. 1, pp. 217-227, 2011.

[4] S. Chowdhury and A. A. Ceder, "Users' willingness to ride an integrated public-transport service: A literature review," Transport Policy, vol. 48, pp. 183-195, 2016.

[5] W. Domschke, "Schedule synchronization for public transit networks," Operations-Research-Spektrum, vol. 11, no. 1, pp. 17-24, 1989.

[6] A. Ceder, B. Golany, and O. Tal, "Creating bus timetables with maximal synchronization," Transportation Research Part A: Policy and Practice, vol. 35, no. 10, pp. 913-928, 2001.

[7] K. Gkiotsalitis, "Bus holding of electric buses with scheduled charging times," IEEE Transactions on Intelligent Transportation Systems, 2020.

[8] P. Rietveld, F. R. Bruinsma, and D. J. Van Vuuren, "Coping with unreliability in public transport chains: A case study for netherlands," Transportation Research Part A: Policy and Practice, vol. 35, no. 6, pp. 539-559, 2001.

[9] K. Gkiotsalitis, O. A. Eikenbroek, and O. Cats, "Robust network-wide bus scheduling with transfer synchronizations," IEEE transactions on intelligent transportation systems, 2019.

[10] C. Fleurent, R. Lessard, and L. Séguin, "Transit timetable synchronization: Evaluation and optimization," in Proceedings of the 9th International Conference on Computer-Aided Scheduling of Public Transport (CASPT). San Diego, California, 2004.
[11] O. J. Ibarra-Rojas and Y. A. Rios-Solis, "Synchronization of bus timetabling," Transportation Research Part B: Methodological, vol. 46, no. 5, pp. 599-614, 2012.

[12] J. Wu, M. Liu, H. Sun, T. Li, Z. Gao, and D. Z. Wang, "Equity-based timetable synchronization optimization in urban subway network," Transportation Research Part C: Emerging Technologies, vol. 51, pp. $1-18,2015$.

[13] J. Parbo, O. A. Nielsen, and C. G. Prato, "User perspectives in public transport timetable optimisation," Transportation Research Part C: Emerging Technologies, vol. 48, pp. 269-284, 2014.

[14] T. Liu and A. A. Ceder, "Integrated public transport timetable synchronization and vehicle scheduling with demand assignment: A biobjective bi-level model using deficit function approach," Transportation research procedia, vol. 23, pp. 341-361, 2017.

[15] Q. Chen, E. Adida, and J. Lin, "Implementation of an iterative headway-based bus holding strategy with real-time information," Public Transport, vol. 4, no. 3, pp. 165-186, 2013.

[16] S. Zolfaghari, N. Azizi, and M. Y. Jaber, "A model for holding strategy in public transit systems with real-time information," International Journal of Transport Management, vol. 2, no. 2, pp. 99-110, 2004.

[17] K. Gkiotsalitis, "A model for the periodic optimization of bus dispatching times," Applied Mathematical Modelling, 2020.

[18] M. M. Nesheli and A. A. Ceder, "Optimal combinations of selected tactics for public-transport transfer synchronization," Transportation Research Part C: Emerging Technologies, vol. 48, pp. 491-504, 2014.

[19] — - "Improved reliability of public transportation using real-time transfer synchronization," Transportation Research Part C: Emerging Technologies, vol. 60, pp. 525-539, 2015.

[20] M. M. Nesheli, A. A. Ceder, and T. Liu, "A robust, tactic-based, real-time framework for public-transport transfer synchronization," Transportation Research Procedia, vol. 9, pp. 246-268, 2015.

[21] K. Gkiotsalitis and N. Maslekar, "Towards transfer synchronization of regularity-based bus operations with sequential hill-climbing," Public transport, vol. 10, no. 2, pp. 335-361, 2018.

[22] R. C. Wong, T. W. Yuen, K. W. Fung, and J. M. Leung, "Optimizing timetable synchronization for rail mass transit," Transportation Science, vol. 42, no. 1, pp. 57-69, 2008.

[23] S. J. Berrebi, K. E. Watkins, and J. A. Laval, "A real-time bus dispatching policy to minimize passenger wait on a high frequency route," Transportation Research Part B: Methodological, vol. 81, pp. 377-389, 2015.

[24] K. Gkiotsalitis and O. Cats, "Multi-constrained bus holding control in time windows with branch and bound and alternating minimization," Transportmetrica B: Transport Dynamics, vol. 7, no. 1, pp. 1258$1285,2019$.

[25] K. Gkiotsalitis and E. Van Berkum, "An exact method for the bus dispatching problem in rolling horizons," Transportation Research Part C: Emerging Technologies, vol. 110, pp. 143-165, 2020.

[26] K. Gkiotsalitis and O. Cats, "Reliable frequency determination: Incorporating information on service uncertainty when setting dispatching headways," Transportation Research Part C: Emerging Technologies, vol. 88, pp. 187-207, 2018.

[27] Y. Xuan, J. Argote, and C. F. Daganzo, "Dynamic bus holding strategies for schedule reliability: Optimal linear control and performance analysis," Transportation Research Part B: Methodological, vol. 45, no. 10, pp. 1831-1845, 2011.

[28] E. R. Randall, B. J. Condry, M. Trompet, and S. K. Campus, "International bus system benchmarking: Performance measurement development, challenges, and lessons learned," in Transportation Research Board 86th Annual Meeting, 21st-25th january, 2007.

[29] M. Trompet, X. Liu, and D. J. Graham, "Development of key performance indicator to compare regularity of service between urban bus operators," Transportation research record, vol. 2216, no. 1, pp. $33-41,2011$.

[30] X. J. Eberlein, N. H. Wilson, and D. Bernstein, "The holding problem with real-time information available," Transportation science, vol. 35, no. 1 , pp. $1-18,2001$.

[31] C. Helmberg, F. Rendl, R. J. Vanderbei, and H. Wolkowicz, "An interior-point method for semidefinite programming," SIAM Journal on Optimization, vol. 6, no. 2, pp. 342-361, 1996.

[32] A. W. Roberts, "Convex functions," in Handbook of Convex Geometry. Elsevier, 1993, pp. 1081-1104.

[33] K. Nederland. (2019) Keolis Timetables. [Online]. Available: https://reizen.keolis.nl/nl/allgo/lijnen 